\title{
Enhanced XAO: the ontology of Xenopus anatomy and development underpins more accurate annotation of gene expression and queries on Xenbase
}

Erik Segerdell ${ }^{*}$, Virgilio G Ponferrada², Christina James-Zorn², Kevin A Burns², Joshua D Fortriede², Wasila M Dahdul ${ }^{3,4}$, Peter D Vize ${ }^{5}$ and Aaron M Zorn²

\begin{abstract}
Background: The African clawed frogs Xenopus laevis and Xenopus tropicalis are prominent animal model organisms. Xenopus research contributes to the understanding of genetic, developmental and molecular mechanisms underlying human disease. The Xenopus Anatomy Ontology (XAO) reflects the anatomy and embryological development of Xenopus. The XAO provides consistent terminology that can be applied to anatomical feature descriptions along with a set of relationships that indicate how each anatomical entity is related to others in the embryo, tadpole, or adult frog. The XAO is integral to the functionality of Xenbase (http://www. xenbase.org), the Xenopus model organism database.

Results: We significantly expanded the XAO in the last five years by adding 612 anatomical terms, 2934 relationships between them, 640 synonyms, and 547 ontology cross-references. Each term now has a definition, so database users and curators can be certain they are selecting the correct term when specifying an anatomical entity. With developmental timing information now asserted for every anatomical term, the ontology provides internal checks that ensure high-quality gene expression and phenotype data annotation. The XAO, now with 1313 defined anatomical and developmental stage terms, has been integrated with Xenbase expression and anatomy term searches and it enables links between various data types including images, clones, and publications. Improvements to the XAO structure and anatomical definitions have also enhanced cross-references to anatomy ontologies of other model organisms and humans, providing a bridge between Xenopus data and other vertebrates. The ontology is free and open to all users.
\end{abstract}

Conclusions: The expanded and improved XAO allows enhanced capture of Xenopus research data and aids mechanisms for performing complex retrieval and analysis of gene expression, phenotypes, and antibodies through text-matching and manual curation. Its comprehensive references to ontologies across taxa help integrate these data for human disease modeling.

Keywords: Anatomy, Bioinformatics, Data annotation, Developmental biology, Embryology, Model organism database, Ontology, Xenopus

\footnotetext{
* Correspondence: segerdel@ohsu.edu

${ }^{1}$ Knight Cancer Institute, Oregon Health \& Science University, Portland, OR, USA

Full list of author information is available at the end of the article
} 


\section{Background}

The embryological literature for Xenopus, the African clawed frog, reaches back more than a century [1]. As many forms of human disease are associated with defects in genes involved in the earliest steps of embryonic development, studying the orthologous genes in Xenopus laevis and $X$. tropicalis as model systems to elucidate the molecular and cellular pathways through which these genes function has grown in strength in recent decades. Annotation and assembly of the Xenopus tropicalis genome demonstrated that it has long regions in which genes exhibit remarkable synteny with the human genome [2], establishing it as an important model for comparative genomics and modeling human gene function. Despite being allotetraploid, X. laevis also reflects genetic synteny to humans with full chromosomal duplication of $X$. tropicalis gene sequences. The $X$. laevis genome is currently being assembled and annotated [3]. In addition to being excellent genetic models, both frog species have large externally developing embryos with rapid embryogenesis, allowing easy study of early vertebrate development from fertilization through organogenesis and limb development. Likewise, large experimentally malleable oocytes, particularly from $X$. laevis, are a key tool in studies of ion channel physiology and toxicology and the cell cycle [4]. Oocytes and synchronously developing embryos are easily obtained in large numbers allowing researchers to quickly gather large amounts of data. Together these two Xenopus species accelerate our understanding of the mechanisms underlying human health and disease [5], yet a daunting challenge remains: to organize, integrate, and make accessible vast quantities of information as it emerges. Xenbase (http://www.xenbase.org), the Xenopus biology and genomics database [6,7], integrates diverse data from high-throughput screens, scientific literature, and other databases (such as NCBI) into a number of database modules, thus allowing researchers to investigate specific genes using well-defined terminologies that bridge different kinds of data. To this end, the Xenopus Anatomy Ontology (XAO) was developed as a structured, controlled terminology that 1) unites anatomy and development of the vertebrate embryo with the molecular and cellular research findings, 2) enables powerful data searches, and 3) facilitates accurate annotation of research findings. From its inception, we intended the $\mathrm{XAO}$ to be integral to the functionality of Xenbase, and as such the XAO acts as a platform to support automated and manual curation and to power the gene expression search feature.

The XAO provides consistent terms for 1313 anatomical features and developmental stages and draws a detailed conceptual picture of the frog from unfertilized egg to adult. Thousands of relationships between terms describe which tissues are components of other tissues, structures, and anatomical systems, as well as articulating the tissues' developmental lineage. The timing of each feature's embryonic development is framed by references to the community-standard Nieuwkoop and Faber (NF) staging series [8]. The XAO is frequently updated and fine-tuned with an emphasis on completeness for each term, and in response to the evolving areas of Xenopus research. This is essential to making high-quality annotations and robust database queries of Xenopus data with maximal utility to the research community. The implementation of the XAO through Xenbase allows the capture of rich content from the scientific literature and it enables the retrieval and analysis of complex data that have been annotated using the ontology, principally gene expression. Curation of mutant and morphant phenotypes using the XAO is currently in development. The XAO has always been freely available to Xenopus researchers and biomedical ontology developers for use in their projects and we continue to encourage them to provide feedback.

Interrelating the $\mathrm{XAO}$ with different bio-ontologies has been a key to making Xenopus data accessible to the broader scientific and biomedical communities [9], enabling researchers to query across orthogonal biological and human disease databases. We previously reported the initial development of the XAO [10], emphasizing strong representation of embryonic development and interoperability with established species-specific and gross-level anatomy ontologies. In 2009, the XAO was recognized as one of eight exemplar ontologies in the Open Biological and Biomedical Ontologies (OBO) Foundry [11]. We identified at that time several areas of the XAO to be targeted for improvement. It needed expansion to support accurate gene expression curation from the Xenopus literature [12] and many existing terms required descriptions and more comprehensive relationships to other terms. Here we report the progress in pursuit of these goals making Xenbase, with its seamless integration of the XAO, a vital and growing biological and genomics database and making the ontology itself a useful resource for Xenopus researchers.

\section{Results and discussion}

\section{Ontology organization and content}

Anatomical entities are organized in a single classification (is_a) framework in the XAO. Upper-level nodes (e.g., 'compound organ', 'organism subdivision') comprise a structural axis of classification cross-referenced to the Common Anatomy Reference Ontology (CARO) [13], providing interoperability with other model organism anatomy ontologies that use CARO as well as a starting point for classifying Xenopus-specific features. The XAO 
reflects various aspects of biological organization with five other logical relationship types.

Cell types, tissues, structures, and sub-systems are described as being part_of other tissues, structures, and systems. The lineage of tissues in the course of development is represented by develops_from relationships. The timing of their development is indicated by starts_during and ends_during relationships linking them to specific developmental stages based on the normal table of Xenopus development by Nieukoop and Faber [8]. This NF stage series, which has long been the standard in Xenopus research, exists as a sub-ontology within the XAO, with preceded_by relationships delineating the temporal ordering of the 66 component stages.

The ontology's developmental tree begins with the specification of the classical vertebrate primary germ layers ('ectoderm,' 'endoderm', and 'mesoderm') and branches into the tissues and structures comprising the growing embryo and tadpole. Ultimately, these features are placed within 19 major anatomical systems, from the 'alimentary system' to the 'urogenital system'. The XAO's latest release (October 9 , 2013) contains 1313 anatomical and developmental stage terms, 5148 relationships, and 695 cross-references to other ontologies (Table 1). Throughout the course of our work we have taken care that the ontology adheres to the community conventions and best practices recommended by the OBO Foundry [14].

The entry for the 'brain' [XAO:0000010], for example, not only provides a consistent name for this feature and a logical classification as a 'cavitated compound organ', its relationship to the term 'central nervous system' indicates where the brain functions while other relationships indicate that the organ first appears at NF stage 22 from its precursor structure, 'anterior neural tube'.

Table 1 A summary of xenopus anatomy ontology content as of October 9, 2013

\begin{tabular}{lc}
\hline Total terms & 1313 \\
Anatomical entities & 1217 \\
Developmental stages & 96 \\
Definitions & 1313 \\
Synonyms & 843 \\
Relationship types & 6 \\
Relationships & 5148 \\
is_a & 1328 \\
part_of & 859 \\
develops_from & 490 \\
starts_during & 1197 \\
ends_during & 1197 \\
preceded_by & 77 \\
Ontology cross-references & 695 \\
\hline
\end{tabular}

Subsequently, the 'forebrain', 'midbrain', and 'hindbrain', are part_of 'brain'. Further divisions relate more regions and structures as part_of these three main regions of the brain; for example, the 'forebrain' has 16 distinct terms, 'midbrain' has 9 terms, and 'hindbrain' has 13 terms defined by part_of relationships. This finer granularity allows more precise gene expression curation that is demanded by modern research.

\section{Expansion and improvements}

As we began to annotate gene expression reported in the literature and to develop a Xenbase expression search interface (released to the public in 2009) it became clear that usability and annotation quality depend on the ontology having comprehensive sets of terms, definitions, and relationships. Expression queries that include anatomical parameters are designed to draw on relationships in the ontology for their functionality. The Xenbase in-house curation interface restricts terms that can be used for annotations at particular stages based on their developmental timing asserted in the ontology.

Since describing the XAO five years ago, we implemented major improvements, expanding it from 701 to 1313 anatomical and developmental stage entities and fleshing out much of its existing content (Figure 1). The ontology, which initially included textual definitions for only 292 terms, now has a definition for every term in the ontology. In its initial release, the ontology comprised to a large extent a "partonomy", with the principal hierarchical structure depending on which entity each anatomical feature is "a part of" rather than what it is "a type of." It lacked a single classification framework that fosters the development of good, clear definitions. Concurrent with our effort to be definition-complete, we ensure that every term has an is_a parent. Furthermore, the majority of embryonic structures previously lacked

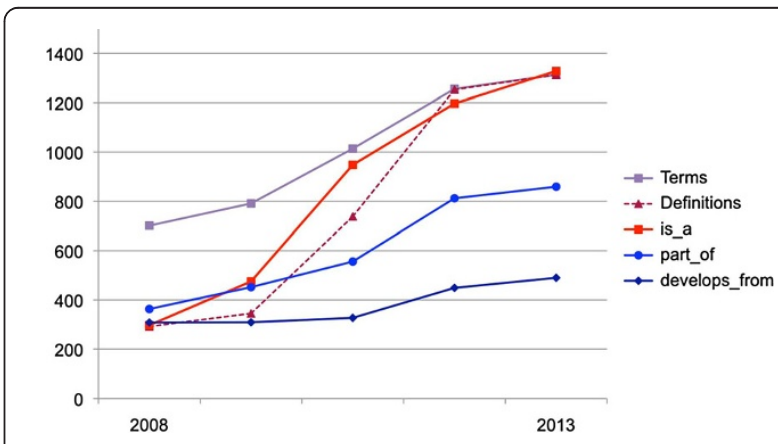

Figure 1 Growth of the xenopus anatomy ontology. In the course of its major public releases since 2008, the number of terms in the ontology has grown by $87 \%$ and the number of part_of and develops_from relationships has substantially increased. The majority of terms in the initial release lacked definitions and is_a parents, while the latest release (October 9, 2013) is definition- and is_a-complete. 
specific starts_during and ends_during stages. All anatomical features now have these relationships following extensive surveys of literature describing various anatomical systems (e.g., the 'skeletal system' [15]). The ontology has grown to contain 843 synonyms (originally 203), 859 part_of relationships (originally 363), and 490 develops_from relationships (originally 308). Now, every anatomical term or one of its is_a ancestors has at least one part_of and at least one develops_from relationship to another term. Information that curators glean from the literature has often led to adjustments of start and end stage relationships in the ontology. Ontologybuilding rules have ensured that the start and end stages associated with related features are consistent and make biological sense. For example, the stage range of 'pronephric mesenchyme', by rule, must be the same as or fall within that of 'mesenchyme', its is_a parent. Similarly, the validity of develops_from and part_of relationships is governed by the timing of the related terms; e.g., if 'pronephric mesenchyme' gives rise to 'pronephric kidney', the latter must appear sometime within or immediately after the former's range of NF stage 21-30 (Figure 2).

By using the starts_during and ends_during developmental stage restrictions we can distinguish transient embryonic structures from tissues that are only present in the adult. Good examples of transient embryonic structures are the 'pronephric kidney' that starts_during NF stage 28 and ends_during NF stage 64, and the 'tail region" that starts_during NF stage 26 and which is resorbed at metamorphosis and thus ends_during 'NF stage 66'. An example of an adult structure is the 'mesonephric kidney', the kidney in the adult frog, that starts_during NF stage 39 and ends_during 'death'. Curators validate the temporal constrains of XAO terms as part of their on going annotation of the published literature and adjust start and end stages accordingly. For example during the recent XAO expansion curators found

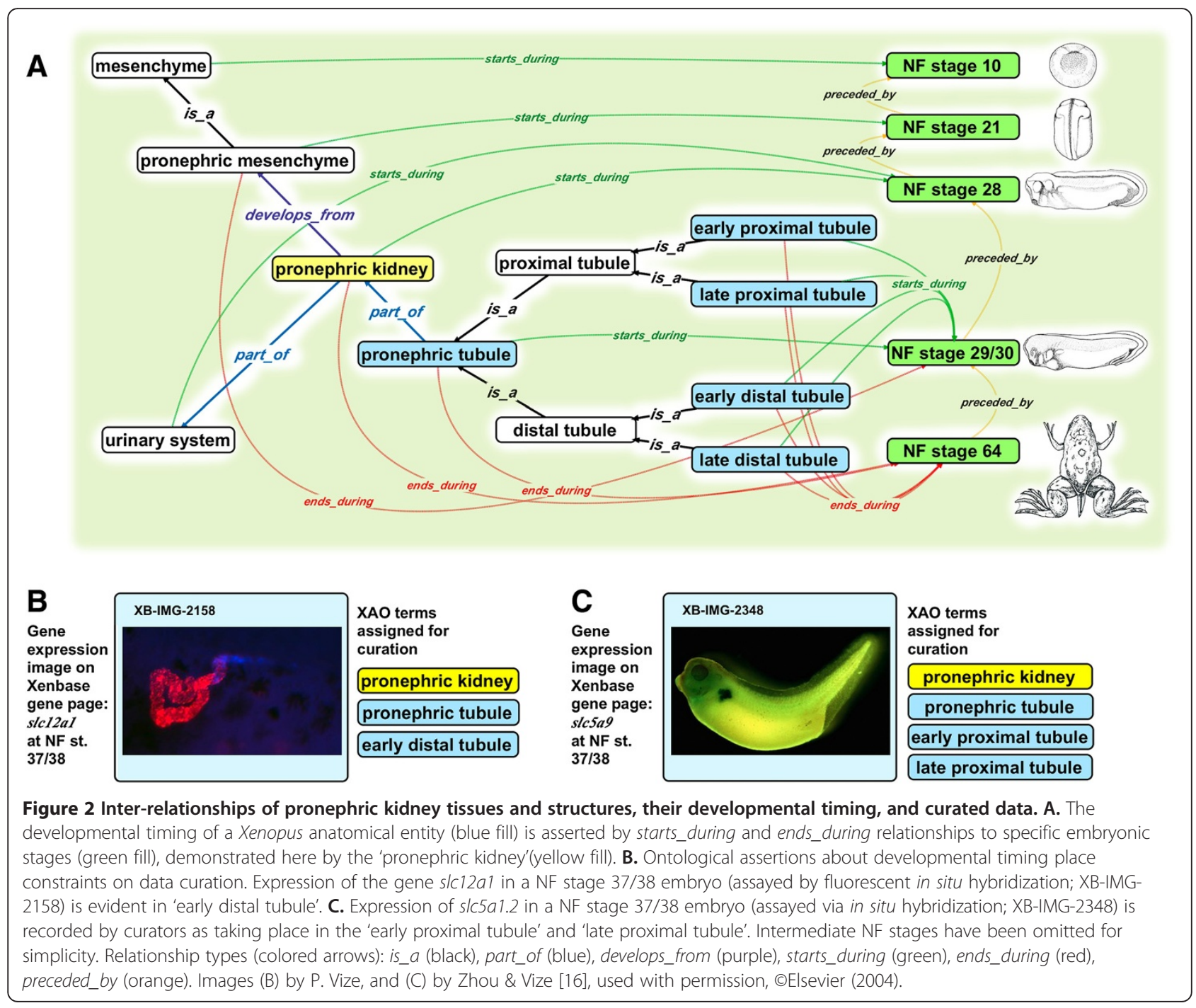


many papers describing the expression of the gene $n k x 2.1$ as one of the earliest markers of lung fate [17-19]. As a result, the starts_during and ends_during for the term 'lung primordia' were revised.

References to other ontologies enable cross-taxon comparisons without the need for complex term translators. The XAO currently contains 695 cross-references; the initial release had only 145 . Its close integration with the cross-species Uber Anatomy Ontology (UBERON) [20] provides a bridge to cell types and other vertebrate anatomy ontologies. The XAO's rich content and referencing has enabled it to be utilized in projects outside of Xenbase, e.g. Bgee [21], which integrates gene expression data from several animal species.

While addressing the XAO's overall condition, we focused on enhancing several specific aspects of the ontology relevant to Xenopus as an animal model:

\section{Musculoskeletal system}

Integration of amphibian limb phenotypes into the Phenoscape Knowledgebase [22], which links evolutionary phenotypes for vertebrates with data from model organisms [23], prompted improvements in this area. The representation of the 'skeletal system', which previously comprised only 35 features, grew to include 146 skeletal element and tissue types based on the Vertebrate Skeletal Anatomy Ontology (VSAO) [24]. Of these, 24 are cranial cartilage terms. The ontology now has 45 individual muscle terms, including many cranial muscles. Only three were described in the original XAO release. In addition, we updated the nomenclature and definitions for limb segments ('autopod,' 'stylopod', and 'zeugopod') and terms for each digit segment and joint regions in support of phenotype curation.

\section{Neural crest}

Xenopus has proven to be a very important model system for studying neural crest (NC) cell differentiation, stem cell properties, epithelial-mesenchymal transition, and cell migration $[25,26]$. In order to support the extensive Xenopus embryo research in this area we have significantly expanded the XAO NC terms. First, Xenbase curators performed an extensive analysis of the NC literature. After drafting preliminary terms, we consulted domain experts from chick, mouse, fish, and frog communities to improve and coordinate neural crest representation in the XAO. Then, in March of 2012 Xenbase staff participated in a cross-ontology RCN NC workshop [27] where we presented our results and made final term modifications to ensure that the XAO NC definitions were in agreement with other ontologies. This exemplifies our general approach to XAO improvement.

Previously, the main 'neural crest' functional domains represented in earlier versions of the XAO included only the 'cranial neural crest', the migrating streams ('mandibular crest', 'hyoid crest', and 'branchial crest') and the 'trunk neural crest'. We expanded this to include 'neural plate border' (with 'pre-chordal neural plate border' and 'chordal neural plate border' domains); 'cardiac neural crest,' 'sacral neural crest', and 'vagal neural crest'; 'premigratory neural crest cell,' 'migratory neural crest cell', and 'postmigratory neural crest cell'; and 'anterior branchial crest' and 'posterior branchial crest' domains. We built develops_from relationships between NC and the tissues and structures to which NC contributes, such as the 'craniofacial skeleton', 'glial cells', and 'enteric neurons' of the 'hindgut', and the 'outflow tract' of the 'heart'.

\section{Neurological structures and stem cells}

While adding 42 more terms for subdivisions and regions of the brain (e.g., 'rhombomere 1' to 'rhombomere 8 ' of 'hindbrain') and 8 new neuron types ('motor neuron', 'interneuron', etc.), the XAO has also doubled the number of neurological placode terms from 10 to 20. These include the 'facial placode', 'glossopharyngeal placode', and 'vagal epibranchial placode' [28]. Stem cell populations of specific regions such as the 'ciliary marginal zone' of the 'retina' have also been added.

\section{Pronephric kidney}

Embryonic kidneys are an important model system for investigating principles regulating multicomponent complex organs [29,30], and Xenopus is prized as a model because of its simplicity and experimental accessibility. Xenbase has a rich complement of annotated expression data for Xenopus 'pronephric kidney' development. The $\mathrm{XAO}$ now contains significant updates to the definitions, timing, relationships, and synonyms of all pronephric structures.

\section{Heart and vasculature}

Xenopus has been instrumental in studies of vertebrate heart development and new transgenic lines are being used to investigate the molecular mechanisms and complex gene regulatory networks underlying human congenital heart defects and diseases [31]. As the anatomy of the developing heart and vasculature is described and imaged in finer detail [32], the XAO must expand to capture this detail. So far we have added 19 new heart terms such as 'primary heart field', 'secondary heart field' and 'epicardial precursor cell', and this will be a continued focus for ontology improvement.

\section{Regenerative structures}

Xenopus has emerged as a leading model for tissue regeneration research [33]. Recent additions of 'blastema' terms specific to the fin, limb, tail, and eye allow 
curators to capture gene expression involved in normal growth and regeneration.

\section{Oogenesis stages}

With their large size, Xenopus oocytes are amenable to studies of ion channels and transporters [34] as well as maternal gene expression. The XAO staging series incorporates seven new unfertilized egg stages, from 'oocyte stage I' to 'mature egg' stage, and oocytes have been added as cell types, enabling curation of gene expression during oogenesis.

\section{Implementation in Xenbase}

Integration of the XAO in Xenbase allows the research community to reap practical benefits without needing expert knowledge of ontologies and their design. It also provides important use cases for how the ontology can be used in research applications in general. The gene expression search interface on Xenbase allows researchers to constrain their queries by a range of stages, narrow queries to a single stage, and/or select anatomy search terms from an autocomplete menu, checkbox panel, or expandable tree. It provides an option to include developmental successor or precursor tissues as search parameters. For example, one can perform an expansive search including data for all derivatives of 'neural crest'.
Behind the scenes, the database leverages the is $a$, part_of, and develops_from assertions in the XAO to retrieve data annotated not only with the exact selected term(s) but with related ones as well. This is a significant improvement over a general text-matching search of Xenbase content, in which a search for 'heart', for example, would only look for that precise phrase and might fail to retrieve data annotated with a more specific heart component such as 'endocardium'. Searching Xenbase with the term 'pronephros' provides another use case: of the 203 image records returned by an ontology-based search for 'pronephric kidney', 25 are not annotated with that exact term and are instead annotated with 'pronephric duct', 'nephrostome', etc. Synonyms in the ontology, furthermore, enable users to choose a search term based on their preferred nomenclature and reduce the chances of an empty result.

A complementary gene expression tool called XenMARK is also available on the web [35]. XenMARK uses an ontology-free annotation system that displays expression images as heat map diagrams projected on embryo schematics. Gene expression queries in XenMARK use coarse-grained 19-term anatomy tags (e.g., 'eye' and 'retina'), thus in XenMARK end users need no detailed knowledge of anatomy terms, but can find genes expressed in the same 'geographical' area of an embryo.

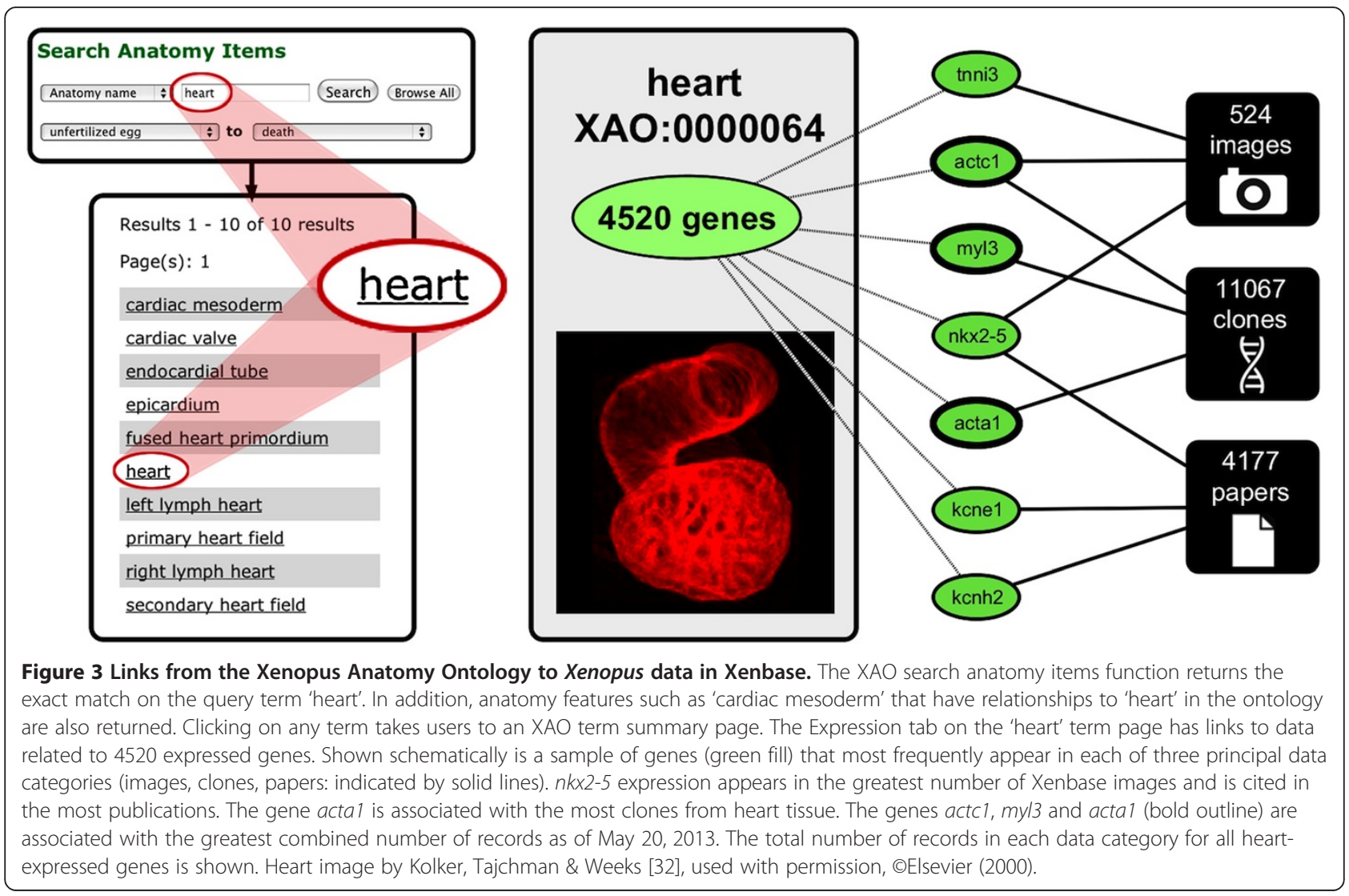


In contrast, the XAO contains over a thousand precise terms (e.g., 42 terms that are part_of the eye with a subset of 23 terms that are part_of the retina) so Xenbase users can perform advanced fine-grained expression queries. Xenbase and XenMARK have data sharing agreements and reciprocal links to enable users to move between the sites easily.

To this end, Xenbase provides anatomy and stage term searches [36], taking users to dedicated term summaries, listing term metadata and links to related Xenopus information. The Expression tab on every anatomy term page (e.g. 'heart' [37]) contains links to data related to genes expressed in that tissue or structure, including images with captions, clones, and publication records (Figure 3). Xenbase complements manually curated data with automated and semi-automated annotation processes [6]. The text-mining tool Textpresso [38] processes newly loaded research article metadata from PubMed [39] and captures terms that match anatomy features, thereby providing links from titles, abstracts, and figure captions to XAO terminology.

\section{Future directions}

In the next phase of XAO development we plan to employ the MIREOT (Minimum Information to Reference an External Ontology Term) [40] technique to import upper ontology terms and cell types, replacing our heretofore manual approach, and to make the XAO compliant with the Basic Formal Ontology [41]. We intend to import a "slim" version of the Gene Ontology (GO) [42] cellular component hierarchy, which will allow us to curate gene expression with cellular localization terms. We are currently testing phenotype curation using combined XAO, GO, and Phenotypic Quality Ontology (PATO) [43] terms.

\section{Conclusions}

Thanks to major expansions and improvements, the $\mathrm{XAO}$ allows capture of richer content from Xenopusspecific scientific literature and research data and provides an essential mechanism for performing complex data retrieval and analysis. We will continue to expand and refine it and to closely interrelate it with other ontologies. The XAO is already essential to gene expression annotation and searches, which will allow researchers to benefit from its further improvement. Xenbase, with an enhanced antibody search function and phenotype database feature currently in development, will continue to drive ontology development. The XAO provides an integral component of entityquality (EQ) and entity-quality-entity (EQE) annotations made in combination with PATO in order to describe phenotypes. While serving these many functions, the ontology's comprehensive references to bio-ontologies across taxa will help integrate data that provide insight into human disease, further enhancing the standing of Xenopus as an important animal model.

\section{Methods}

The Xenopus Anatomy Ontology is free and open to all users. It may be downloaded from the Xenbase FTP site [44] in file formats that allow it to be opened in two freely available tools, OBO-Edit [45] and Protégé [46]. An OBO-compliant version is available at the OBO Foundry [47] and in Google Projects [48]. Users may also browse the ontology at Xenbase [49] and at a variety of external informatics sites such as Ontobee [50].

Xenbase employs an in-house system of shared documents and spreadsheets where curators request new terms with metadata (definitions, relationships, and stages) and discuss wider structural changes. Public requests and feedback may be submitted at the XAO SourceForge [51] and Google Projects issue trackers.

We regularly seek expertise and input from developers of other vertebrate anatomy ontologies (e.g., the Amphibian Anatomy Ontology [52] and Zebrafish Anatomy Ontology [53]) and the Uber Anatomy Ontology. The $\mathrm{XAO}$ adheres to a comprehensive referencing (xref) scheme. This consists of CARO xrefs for upper-level terms and Cell Type (CL) [54] or UBERON xrefs for other applicable terms (the Amphibian Anatomy Ontology, an effort closely related to the Xenopus ontology, and the VSAO have recently been absorbed by UBERON, so unlike in previous releases, XAO terms now refer to the relevant UBERON entries and not the original ontologies). We strive to make definitions consistent with UBERON, other anatomy ontologies, and the $\mathrm{CL}$, augmenting them as necessary to reflect their specificity to Xenopus.

\section{Competing interests}

The authors declare that they have no competing interests.

\section{Authors' contributions}

ES, VGP, CJZ drafted the manuscript. ES, VGP, CJZ, KAB, JDF and WMD developed and updated the ontology. PDV and AMZ supervised the project and oversaw XAO implementation. All authors read and approved the final manuscript.

\section{Acknowledgements}

Xenbase and XAO development are funded by the National Institute of Child Health and Human Development grant P41 HD064556. WMD and CJZ are supported in part by the National Science Foundation for VSAO development, under Grant Numbers DBI-0641025, DBI-1062404, and DBI-1062542, and by the National Evolutionary Synthesis Center under NSF EF-0423641 and NSF \#EF-0905606. Any opinions, findings, and conclusions or recommendations expressed in this material are those of the author(s) and do not necessarily reflect the views of the National Science Foundation. We are grateful to Sally Moody, Samantha Brugmann, and Jean-Pierre Saint-Jeannet for advice on NC terms, and to participants in the Neural Crest Ontology Workshop, sponsored by the Phenotype Research Coordination Network, February 2012, Durham, North Carolina. We thank Chris Mungall and Melissa Haendel for advice on ontology improvements, best practices, and version control, and Melissa Haendel for comments on the manuscript. 


\section{Author details}

${ }^{1}$ Knight Cancer Institute, Oregon Health \& Science University, Portland, OR, USA. ²Division of Developmental Biology, Cincinnati Children's Research Foundation, Cincinnati, OH, USA. ${ }^{3}$ Department of Biology, University of South Dakota, Vermillion, SD, USA. ${ }^{4}$ National Evolutionary Synthesis Center, Durham, NC, USA. ${ }^{5}$ Department of Biological Science, University of Calgary, Calgary, $A B$, Canada.

Received: 22 June 2013 Accepted: 11 October 2013

Published: 18 October 2013

\section{References}

1. Bles EJ: On the breeding habits of Xenopus laevis Daud. Proc Camb Phil Soc 1901, 11:220-222.

2. Hellsten U, Harland RM, Gilchrist MJ, Hendrix D, Jurka J, Kapitonov V Ovcharenko I, Putnam NH, Shu S, Taher L, Blitz IL, Blumberg B, Dichmann DS, Dubchak I, Amaya E, Detter JC, Fletcher R, Gerhard DS, Goodstein D, Graves T, Grigoriev IV, Grimwood J, Kawashima T, Lindquist E, Lucas SM, Mead PE, Mitros T, Ogino H, Ohta Y, Poliakov AV, Pollet N, Robert J, Salamov A, Sater AK, Schmutz J, Terry A, Vize PD, Warren WC, Wells D, Wills A, Wilson RK, Zimmerman LB, Zorn AM, Grainger R, Grammer T, Khokha MK, Richardson PM, Rokhsar DS: The genome of the Western clawed frog Xenopus tropicalis. Science 2010, 328(5978):633-636

3. Xenopus Genome Project. http://polaris.icmb.utexas.edu/index.php/ Xenopus_Genome_Project.

4. Khokha MK: Xenopus white papers and resources: folding functional genomics and genetics into the frog. Genesis 2012, 50(3):133-142.

5. Grainger RM: Xenopus tropicalis as a model organism for genetics and genomics: past, present, and future. Methods Mol Biol 2012, 917:3-15.

6. Bowes JB, Snyder KA, Segerdell E, Gibb R, Jarabek C, Noumen E, Pollet N, Vize PD: Xenbase: a Xenopus biology and genomics resource. Nucleic Acids Res 2008, 36(Database issue):D761-D767.

7. James-Zorn C, Ponferrada VG, Jarabek CJ, Burns KA, Segerdell EJ, Lee J, Snyder K, Bhattacharyya B, Karpinka JB, Fortriede J, Bowes JB, Zorn AM, Vize PD: Xenbase: expansion and updates of the Xenopus model organism database. Nucleic Acids Res 2013, 41(Database issue):D865-D870.

8. Nieuwkoop PD, Faber J: Normal Table of Xenopus laevis (Daudin). New York: Garland Publishing; 1994.

9. Noy NF, Shah NH, Whetzel PL, Dai B, Dorf M, Griffith $N$, Jonquet C, Rubin DL, Storey MA, Chute CG, Musen MA: BioPortal: ontologies and integrated data resources at the click of a mouse. Nucleic Acids Res 2009, 37(Web Server issue):W170-W173.

10. Segerdell E, Bowes JB, Pollet N, Vize PD: An ontology for Xenopus anatomy and development. BMC Dev Biol 2008, 8:92.

11. The Open Biological and Biomedical Ontologies. http://www.obofoundry.org

12. Bowes JB, Snyder KA, James-Zorn C, Ponferrada VG, Jarabek CJ, Burns KA, Bhattacharyya B, Zorn AM, Vize PD: The Xenbase literature curation process. Database 2013, 2013:bas046.

13. Haendel MA, Neuhaus F, Osumi-Sutherland D, Mabee PM, Mejino JLV Jr, Mungall CJ, Smith B: CARO-The Common Anatomy Reference Ontology. In Anatomy Ontologies for Bioinformatics. Edited by Burger A, Davidson D, Baldock R. New York: Springer; 2007:311-333.

14. Smith B, Ashburner M, Rosse C, Bard J, Bug W, Ceusters W, Goldberg L, Eilbeck K, Ireland A, Mungall CJ, Consortium OBI, Leontis N, Rocca-Serra P, Ruttenberg A, Sansone SA, Scheuermann RH, Shah N, Whetzel PL, Lewis S: The OBO Foundry: coordinated evolution of ontologies to support biomedical data integration. Nat Biotechnol 2007, 25:1251-1255.

15. Trueb L, Hanken J: Skeletal development in Xenopus laevis (Anura: Pipidae). J Morphol 1992, 214(1):1-41.

16. Zhou X, Vize PD: Proximo-distal specialization of epithelial transport processes within the Xenopus pronephric kidney tubules. Dev Biol 2004, 271(2):322-338.

17. Small EM, Vokes SA, Garriock RJ, Li D, Krieg PA: Developmental expression of the Xenopus Nkx2-1 and Nkx2-4 genes. Mech Dev 2000, 2:259-262.

18. Hollemann T, Pieler T: Xnkx-2.1: a homeobox gene expressed during early forebrain, lung and thyroid development in Xenopus laevis. Dev Genes Evol 2000, 1:579-581.

19. Rankin SA, Neto A, Gallas A, Gómez-Skarmeta JL, Zorn AM: Suppression of Bmp4 signaling by the zinc-finger repressors Osr1 and Osr2 is required for Wnt/beta-catenin-mediated lung specification in Xenopus. Development 2012, 139:3010-3020.
20. Mungall CJ, Torniai C, Gkoutos GV, Lewis SE, Haendel MA: Uberon, an integrative multi-species anatomy ontology. Genome Biol 2012, 13(1):R5.

21. Bgee: a dataBase for Gene Expression Evolution. http://bgee.unil.ch.

22. Phenoscape. http://www.phenoscape.org

23. Mabee BP, Balhoff JP, Dahdul WM, Lapp H, Midford PE, Vision TJ, Westerfield M: 500,000 Fish phenotypes: the new informatics landscape for evolutionary and developmental biology of the vertebrate skeleton. J Appl Ichthyol 2012, 28(3):300-305.

24. Dahdul WM, Balhoff JP, Blackburn DC, Diehl AD, Haendel MA, Hall BK, Lapp $\mathrm{H}$, Lundberg JG, Mungall CJ, Ringwald M, Segerdell E, Van Slyke CE, Vickaryous MK, Westerfield M, Mabee PM: A unified anatomy ontology of the vertebrate skeletal system. PLoS One 2012, 7(12):e51070.

25. Bronner ME, LeDouarin NM: Development and evolution of the neural crest: an overview. Dev Biol 2012, 366(1):2-9.

26. Mayor R, Theveneau E: The neural crest. Development 2013, 140(11):2247-2251.

27. Phenotype RCN Neural Crest workshop, March 2012. 2012. http://www. phenotypercn.org/?p=1047

28. Schlosser G: Induction and specification of cranial placodes. Dev Biol 2006 294(2):303-351.

29. Vize PD, Seufert DW, Carroll TJ, Wallingford JB: Model systems for the study of kidney development: use of the pronephros in the analysis of organ induction and patterning. Dev Biol 1997, 188(2):189-204.

30. Wessely $\mathrm{O}$, Tran U: Xenopus pronephros development-past, present, and future. Pediatr Nephrol 2011, 26(9):1545-1551.

31. Kaltenbrun E, Tandon P, Amin NM, Waldron L, Showell C, Conlon FL: Xenopus: an emerging model for studying congenital heart disease. Birth Defects Res A Clin Mol Teratol 2011, 91(6):495-510.

32. Kolker SJ, Tajchman U, Weeks DL: Confocal imaging of early heart development in Xenopus laevis. Dev Biol 2000, 218(1):64-73.

33. Beck CW, Izpisúa Belmonte JC, Christen B: Beyond early development: Xenopus as an emerging model for the study of regenerative mechanisms. Dev Dyn 2009, 238(6):1226-1248.

34. Barnard EA, Miledi R, Sumikawa K: Translation of exogenous messenge RNA coding for nicotinic acetylcholine receptors produces functional receptors in Xenopus oocytes. Proc R Soc Lond B Biol Sci 1982, 215(1199):241-246.

35. Gilchrist MJ, Christensen MB, Bronchain O, Brunet F, Chesneau A, Fenger U, Geach TJ, Ironfield HV, Kaya F, Kricha S, Lea R, Massé K, Néant I, Paillard E, Parain K, Perron M, Sinzelle L, Souopgui J, Thuret R, Ymlahi-Ouazzani Q, Pollet N: Database of queryable gene expression patterns for Xenopus. Dev Dyn 2009, 238(6):1379-1388.

36. Xenbase: Search Anatomy Items. http://www.xenbase.org/anatomy/anatomy. do?method=display\&tabld $=2$

37. Heart expression [Xenopus] - Xenbase XAO Anatomy Item. http://www. xenbase.org/anatomy/geneExpression.do?method=display\& anatomyltemld=63\&anatomy Name=heart\&beginlndex=1\&endlndex= $100 \&$ orderBy $=4 \&$ tabld $=2$

38. Textpresso. http://www.textpresso.org.

39. PubMed - NCBI. http://www.ncbi.nlm.nih.gov/pubmed.

40. Courtot M, Gibson F, Lister AL, Malone J, Schober D, Brinkman RR, Ruttenberg A: MIREOT: the minimum information to reference an external ontology term. Appl Ontol 2011, 6:23-33.

41. Grenon P, Smith B, Goldberg L: Biodynamic Ontology: applying BFO in the Biomedical Domain. In Ontologies in Medicine. Edited by Pisanelli DM. Amsterdam: IOS Press; 2004:20-38.

42. Ashburner M, Ball CA, Blake JA, Botstein D, Butler H, Cherry JM, Davis AP, Dolinski K, Dwight SS, Eppig JT, Harris MA, Hill DP, Issel-Tarver L, Kasarskis A, Lewis S, Matese JC, Richardson JE, Ringwald M, Rubin GM, Sherlock G: Gene ontology: tool for the unification of biology. The gene ontology consortium. Nat Genet 2000, 25(1):25-29.

43. Ontology Detail: Phenotypic quality. http://www.obofoundry.org/cgi-bin/ detail.cgi?id=quality.

44. Xenbase FTP Xenopus Anatomy Ontology. ftp://ftp.xenbase.org/pub/ XenopusAnatomyOntology.

45. OBO-Edit. http://oboedit.org.

46. The Protégé Ontology Editor and Knowledge Acquisition System. http://protege.stanford.edu.

47. Ontology Detail: Xenopus anatomy and development. http://www. obofoundry.org/cgi-bin/detail.cgi?id=xenopus_anatomy.

48. xenopus-anatomy-ontology_Google Project Housing. http://code.google. $\mathrm{com} / \mathrm{p} /$ xenopus-anatomy-ontology. 
49. Xenopus Anatomical Ontology—Xenbase. http://www.xenbase.org/anatomy/ xao.do?method=display.

50. Ontobee: XAO. http://www.ontobee.org/browser/index.php?o=XAO.

51. Open Biomedical Ontologies / Xenopus Anatomy (XAO) term requests. http://sourceforge.net/p/obo/xenopus-anatomy-xao-term-requests/.

52. Maglia AM, Leopold JL, Pugener LA, Gauch S: An anatomical ontology of amphibians. Pac Symp Biocomput 2007, 12:367-378.

53. Spraque J, Bayraktaroglu L, Bradford Y, Conlin T, Dunn N, Fashena D, Frazer K, Haendel M, Howe DG, Knight J, Mani P, Moxon SA, Pich C, Ramachandran S, Schaper K, Segerdell E, Shao X, Singer A, Song P, Sprunger B, Van Slyke

$C E$, Westerfield M: The Zebrafish Information Network: the zebrafish model organism database provides expanded support for genotypes and phenotypes. Nucleic Acids Res 2008, 36:D768-D772.

54. Cellontology.org. http://cellontology.org.

doi:10.1186/2041-1480-4-31

Cite this article as: Segerdell et al:: Enhanced XAO: the ontology of Xenopus anatomy and development underpins more accurate annotation of gene expression and queries on Xenbase. Journal of Biomedical Semantics 2013 4:31.

\section{Submit your next manuscript to BioMed Central and take full advantage of:}

- Convenient online submission

- Thorough peer review

- No space constraints or color figure charges

- Immediate publication on acceptance

- Inclusion in PubMed, CAS, Scopus and Google Scholar

- Research which is freely available for redistribution 